\title{
About division by 1
}

\author{
Alain Lascoux* \\ IGM, C.N.R.S \\ 77454 Marne La Vallée Cedex 2 FRANCE \\ Alain.Lascoux@univ-mlv.fr
}

Submitted: May 1, 2001; Accepted: September 19, 2001.

MR Subject Classifications: 05E05, 11A05

\begin{abstract}
The Euclidean division of two formal series in one variable produces a sequence of series that we obtain explicitly, remarking that the case where one of the two initial series is 1 is sufficiently generic. As an application, we define a Wronskian of symmetric functions.
\end{abstract}

The Euclidean division of two polynomials $P(z), Q(z)$, in one variable $z$, of consecutive degrees, produces a sequence of linear factors (the successive quotients), and a sequence of successive remainders, both families being symmetric functions in the roots of $P$ and $Q$ separately.

Euclidean division can also be applied to formal series in $z$, but it never stops in the generic case, leaving time enough to observe the law of the coefficients appearing in the process.

Moreover, since the quotient of two formal series is also a formal series, it does not make much difference if we suppose that one of the two initial series is 1 . This renders the division of series simpler than that of polynomials; in fact the latter could be obtained from the former.

By formal series we mean a unitary series

$$
f(z)=1+c_{1} z+c_{2} z^{2}+\cdots .
$$

We shall moreover formally factorize it

$$
f(z)=\sigma_{z}(\mathbb{A}):=\prod_{a \in \mathbb{A}}(1-z a)^{-1}=\sum_{i=0}^{\infty} z^{i} S_{i}(\mathbb{A}),
$$

${ }^{*}$ Written during the conference Applications of the Macdonald Polynomials, at the Newton Institute in April 2001. 
the alphabet $\mathbb{A}$ being supposed to be an infinite set of indeterminates, or of complex numbers, the coefficients $S_{i}(\mathbb{A})$ being called the complete functions in $\mathbb{A}$.

Given two series, dividing $f_{-1}(z)=\sigma_{z}(\mathbb{A})$ by $f_{0}(z)=\sigma_{z}(\mathbb{B})$ means finding the unique coefficients $\alpha, \beta$ such that

$$
\left(\sigma_{z}(\mathbb{A})-(1+\alpha z) \sigma_{z}(\mathbb{B})\right) \frac{1}{\beta} z^{-2}
$$

is a unitary series $f_{1}(z)=\sigma_{z}(\mathbb{C})$.

Dividing in turn $f_{0}(z)$ by $f_{1}(z)$, one obtains $f_{2}(z)$, and iterating one gets from the initial pair $\left(f_{-1}, f_{0}\right)$ an infinite sequence of series $f_{-1}, f_{0}, f_{1}, f_{2}, f_{3}, \ldots$, calling $f_{k}$ the $k$-th remainder.

However, all the equations

$$
f_{k-1}(z)=\left(1+\alpha_{k} z\right) f_{k}(z)+\beta_{k} z^{2} f_{k+1}(z)
$$

can be divided by $f_{0}(z)=\sigma_{z}(\mathbb{B})$. If the $k$-th remainder for the pair $\left(\sigma_{z}(\mathbb{A}), \sigma_{z}(\mathbb{B})\right)$ is $\sigma_{z}(\mathbb{C})$, then the $k$-th remainder for the pair $\left(\sigma_{z}(\mathbb{A}) / \sigma_{z}(\mathbb{B}), 1\right)$ is

$$
\sigma_{z}(\mathbb{C}-\mathbb{B})=\sum_{i=0}^{\infty} z^{i} S_{i}(\mathbb{C}-\mathbb{B}):=\frac{\prod_{b \in \mathbb{B}}(1-z b)}{\prod_{c \in \mathbb{C}}(1-z c)} .
$$

Indeed, already at the first step, one sees that the equation

$$
\sigma_{z}(\mathbb{A})=\left(1+z S_{1}(\mathbb{A}-\mathbb{B})\right) \sigma_{z}(\mathbb{B})+S_{2}(\mathbb{A}-\mathbb{B}) z^{2} f_{1}(z)
$$

is equivalent to

$$
\sigma_{z}(\mathbb{A}-\mathbb{B})=\left(1+z S_{1}(\mathbb{A}-\mathbb{B})\right) 1+S_{2}(\mathbb{A}-\mathbb{B}) z^{2} f_{1}(z) / \sigma_{z}(\mathbb{B})
$$

since the two coefficients are functions of $\mathbb{A}-\mathbb{B}$.

Of course equation (4) expands into

$$
\sigma_{z}(\mathbb{A}-\mathbb{B})=\left(1+z S_{1}(\mathbb{A}-\mathbb{B})\right)+S_{2}(\mathbb{A}-\mathbb{B}) z^{2} \sum_{i} z^{i} \frac{S_{2+i}(\mathbb{A}-\mathbb{B})}{S_{2}(\mathbb{A}-\mathbb{B})}
$$

We shall see that the other remainders can be as easily written. We just need to recall the definition of a Schur function $S_{\lambda}(\mathbb{A}-\mathbb{B})$, where $\lambda=\left[\lambda_{1} \geq \lambda_{2} \geq \cdots \geq \lambda_{\ell} \geq 0\right]$ is a partition :

$$
S_{\lambda}(\mathbb{A}-\mathbb{B})=\operatorname{det}\left(S_{\lambda_{i}+j-i}(\mathbb{A}-\mathbb{B})\right)_{1 \leq i, j \leq \ell}
$$

Then

Theorem 1 The $k$-th remainder in the Euclidean division of $\sigma_{z}(\mathbb{A})$ by 1 is

$$
f_{k}(z)=S_{(k+1)^{k}}(\mathbb{A})^{-1} \sum_{i=0}^{\infty} z^{i} S_{k+1+i,(k+1)^{k-1}}(\mathbb{A}) .
$$


The $k$-th remainder of the division of $\sigma_{z}(\mathbb{A})$ by $\sigma_{z}(\mathbb{B})$ is

$$
f_{k}(z)=S_{(k+1)^{k}}(\mathbb{A}-\mathbb{B})^{-1} \sigma_{z}(\mathbb{B}) \sum_{i=0}^{\infty} z^{i} S_{k+1+i,(k+1)^{k-1}}(\mathbb{A}-\mathbb{B}) .
$$

Proof. Merging all equations (2) together, one can characterize in the first case $f_{k}(z)$ as the unique series such that there exists scalars

$$
\begin{gathered}
\alpha_{1}^{(k)}, \ldots, \alpha_{k-1}^{(k)}, \beta_{1}^{(k)}, \ldots, \beta_{k}^{(k)}, \gamma^{(k)}: \\
z^{2 k} \gamma^{(k)} f_{k}(z)=\left(1+\alpha_{1}^{(k)} z+\cdots+\alpha_{k-1}^{(k)} 2 z^{k-1}\right) \sigma_{z}(\mathbb{A})-\left(1+\beta_{1}^{(k)} z+\cdots+\beta_{k}^{(k)} z^{k}\right) 1 .
\end{gathered}
$$

Defining

I claim that

$$
\left.\sigma_{y}\left(\mathbb{A} \pm \frac{1}{z}\right)=\sigma_{y}(\mathbb{A}) \sigma_{y}\left( \pm \frac{1}{z}\right)\right)=\sigma_{y}(\mathbb{A})(1-y / z)^{\mp 1},
$$

$$
z^{k-1} S_{(k+1)^{k-1}}\left(\mathbb{A}-\frac{1}{z}\right) \sigma_{z}(\mathbb{A})+(-z)^{k} S_{k^{k}}\left(\mathbb{A}+\frac{1}{z}\right)
$$

is equal to $z^{2 k} S_{(k+1)^{k}}(\mathbb{A})$ modulo terms of higher degree in $z$.

Indeed, $z^{k-1} S_{(k+1)^{k-1}}(\mathbb{A}-1 / z) \sigma_{z}(\mathbb{A})$ can be written as the $k \times k$ determinant

$$
\left|\begin{array}{cccc}
z^{k-1} \sigma_{z}(\mathbb{A}) & z^{k-2} \sigma_{z}(\mathbb{A}) & \cdots & \sigma_{z}(\mathbb{A}) \\
S_{k}(\mathbb{A}) & S_{k+1}(\mathbb{A}) & \cdots & S_{2 k-1}(\mathbb{A}) \\
\vdots & \vdots & & \vdots \\
S_{2}(\mathbb{A}) & S_{3}(\mathbb{A}) & \cdots & S_{k+1}(\mathbb{A})
\end{array}\right|
$$

because, subtracting from each column, except the first, $1 / z$ times the preceding column and using $S_{j}(\mathbb{A}-1 / z)=S_{j}(\mathbb{A})-S_{j-1}(\mathbb{A}) / z, j \in \mathbb{Z}$, the determinant factorizes into $z^{k-1} \sigma_{z}(\mathbb{A}) S_{(k+1)^{k-1}}(\mathbb{A}-1 / z)$.

The coefficients of $z^{k+1}, \ldots, z^{2 k-1}$ are the functions $S_{2,(k+1)^{k-1}}(\mathbb{A}), \ldots, S_{k,(k+1)^{k-1}}(\mathbb{A})$ which are zero, having two identical rows in their determinantal expression.

The coefficients of $z^{0}, \ldots, z^{k}$ are

$$
S_{1-k,(k+1)^{k-1}}(\mathbb{A}), \ldots, S_{1,(k+1)^{k-1}}(\mathbb{A})
$$

which, after permuting their first row with the others, are recognized to be

$$
S_{k^{k-1}, 0}(\mathbb{A}), \ldots, S_{k^{k-1}, k}(\mathbb{A}),
$$

up to the sign $(-1)^{k-1}$.

These terms sum up to $(-z)^{k} S_{k^{k}}(\mathbb{A}+1 / z)$, and the required series $f_{k}(z)$ is obtained by dividing by $(-1)^{k-1} S_{k^{k-1}}(\mathbb{A})$.

QED

The first defining equations for the $f_{i}$ 's are, writing $S_{j}$ for $S_{j}(\mathbb{A})$ :

$$
z^{2} S_{2} f_{1}=\sigma_{z}(\mathbb{A})-\left(1+z S_{1}\right)=\sigma_{z}(\mathbb{A})-z S_{1}\left(\mathbb{A}+\frac{1}{z}\right),
$$




$$
\begin{gathered}
-z^{4} S_{33} / S_{2} f_{2}=\left(1-z S_{3} / S_{2}\right) \sigma_{z}(A)-\left(1+z S_{21} / S_{2}+z^{2} S_{22} / S_{2}\right)= \\
=-S_{3}\left(\mathbb{A}-\frac{1}{z}\right) \sigma_{z}(\mathbb{A}) / S_{2}-S_{22}\left(\mathbb{A}+\frac{1}{z}\right) / S_{2} \\
z^{6} S_{444} / S_{33} f_{3}=\left(1-z S_{43} / S_{33}+z^{2} S_{44} / S_{33}\right) \sigma_{z}(\mathbb{A})-\left(1+z S_{331} / S_{33}+z^{2} S_{332} / S_{33}\right. \\
\left.+z^{3} S_{333} / S_{33}\right)=z^{2} S_{44}\left(\mathbb{A}-\frac{1}{z}\right) \sigma_{z}(\mathbb{A}) / S_{33}-z^{3} S_{333}\left(\mathbb{A}+\frac{1}{z}\right) / S_{33} .
\end{gathered}
$$

Equation (9) can be understood as giving the Padé approximant of degree $[k, k-1]$ of the series $\sigma_{z}(\mathbb{A})$ :

$$
\sigma_{z}(\mathbb{A})=(-1)^{k-1} \frac{z S_{k^{k}}(\mathbb{A}+1 / z)}{S_{(k+1)^{k-1}}(\mathbb{A}-1 / z)}+z^{2 k} \frac{S_{(k+1)^{k}}(\mathbb{A})}{z^{k-1} S_{(k+1)^{k-1}}(\mathbb{A}-1 / z)} f_{k}(z) .
$$

Sylvester treated the Euclidean division by a different method, using summations on subsets of roots (cf. [LP]). We shall for our part interpret now the Euclidean division as producing a sequence of alphabets from a given one. This time, it is more convenient to divide 1 by a series, and put $f_{-1}=1=\sigma_{z}(0), f_{0}=\sigma_{z}(\mathbb{A})=\sigma_{z}\left(\mathbb{A}^{0}\right), \ldots, f_{k}=\sigma_{z}\left(\mathbb{A}^{k}\right), \ldots$

From (7) one has

$$
\sigma_{z}\left(\mathbb{A}^{k}-\mathbb{A}^{0}\right)=S_{(k+1)^{k}}\left(0-\mathbb{A}^{0}\right)^{-1} \sum_{i=0}^{\infty} z^{i} S_{k+1+i,(k+1)^{k-1}}\left(0-\mathbb{A}^{0}\right),
$$

that is,

$$
S_{k^{k+1}}(\mathbb{A}) \sigma_{z}\left(\mathbb{A}^{k}\right)=\sigma_{z}(\mathbb{A}) \sum_{i=0}^{\infty} z^{i} S_{k+1+i,(k+1)^{k-1}}(-\mathbb{A})
$$

Proposition 2 The successive remainders $\sigma_{z}\left(\mathbb{A}^{k}\right)$ in the division of 1 by $\sigma_{z}(\mathbb{A})$ satisfy

$$
S_{k^{k+1}}(\mathbb{A}) \sigma_{z}\left(\mathbb{A}^{k}\right)=\sum_{i=0}^{\infty} z^{i} S_{k+i, k^{k}}(\mathbb{A})
$$

Proof. Instead of having recourse to determinants, and using relations between minors, let us invoke symmetrizing operators. Suppose the cardinality of $\mathbb{A}$ to be finite, $\mathbb{A}=$ $\left\{a_{1}, \ldots, a_{N}\right\}$, before letting it tend to infinity. Let $\pi_{\omega}$ be the maximal isobaric divided difference, that is, the operator such that the image of $a^{\lambda}:=a_{1}^{\lambda_{1}} \cdots a_{N}^{\lambda_{N}}$ is $S_{\lambda}(\mathbb{A})$, for any $\lambda \in \mathbb{N}^{N}$. The series on the right of (12) is the image under $\pi_{\omega}$ of

$$
\left(a_{1} \cdots a_{k+1}\right)^{k} /\left(1-z a_{1}\right)
$$

and also of

$$
\left(a_{1} \cdots a_{k+1}\right)^{k} /\left(1-z a_{1}\right) \cdots\left(1-z a_{k+1}\right),
$$


which can be written

$$
\frac{\left(a_{1} \cdots a_{k+1}\right)^{k}\left(1-z a_{k+2}\right) \cdots\left(1-z a_{N}\right)}{\left(1-z a_{1}\right) \cdots\left(1-z a_{N}\right)} .
$$

The denominator is symmetrical in $a_{1}, \ldots, a_{N}$, and thus commutes with $\pi_{\omega}$. As for the numerator, the only monomials giving a non-zero contribution have exponents

$$
\underbrace{k, \ldots, k}_{k+1} \underbrace{1, \ldots, 1}_{i} \underbrace{0, \ldots, 0}_{N-k-1-i}, 0 \leq i \leq N-k-1 .
$$

Therefore

$$
\sum_{i=0}^{\infty} S_{k+i, k^{k}}(\mathbb{A})=\sum(-z)^{i} S_{k^{k+1,1^{i}}}(\mathbb{A}) \sigma_{z}(\mathbb{A}),
$$

which gives $(11)$ since $S_{\lambda}(\mathbb{A})=(-1)^{|\lambda|} S_{\lambda \sim}(-\mathbb{A})$, where $\lambda^{\sim}$ is the conjugate to the partition $\lambda$.

The sequences $\mathbb{A}, \mathbb{A}^{1}, \mathbb{A}^{2}, \ldots$ have been considered by B.Leclerc, to whom the following notion of a Wronskian of complete symmetric functions is due (there are more general Wronskians associated to any set of symmetric functions, and any alphabet).

Let $n$ be a positive integer, $k_{1}, \ldots, k_{n} \in \mathbb{N}$, and $\mathbb{A}$ be an alphabet. Then the Wronskian $W\left(S_{k_{1}}, \ldots, S_{k_{n}} ; \mathbb{A}\right)$ is the determinant

$$
\operatorname{det}\left|S_{k_{j}-i}\left(\mathbb{A}^{i}\right)\right|_{0 \leq i \leq n-1,1 \leq j \leq n}
$$

where $\mathbb{A}^{0}:=\mathbb{A}, \mathbb{A}^{1}, \mathbb{A}^{2} \ldots$ is the sequence of alphabets obtained in the Euclidean division of 1 by $\sigma_{z}(\mathbb{A})$.

As an application of his study of relations between minors [Bl], B. Leclerc obtained in an unpublished note :

Theorem 3 Let $n$ be a positive integer, $K=\left[k_{1}, \ldots, k_{n}\right] \in \mathbb{N}^{n}$, $\mathbb{A}$ be an alphabet. Then

$$
W\left(S_{k_{1}}, \ldots, S_{k_{n}} ; \mathbb{A}\right)=S_{K+[n-1, \ldots, 0]}(\mathbb{A}) / S_{(n-1)^{n}}(\mathbb{A})
$$

Proof. To simplify the notation, we shall take $n=4, K=[\alpha, \beta, \gamma, \delta] "$. Then the Wronskian, in terms of functions of $\mathbb{A}$ only, is, according to (12),

$$
\left|\begin{array}{llll}
S_{\alpha 000} & S_{\beta 000} & S_{\gamma 000} & S_{\delta 000} \\
S_{\alpha 100} & S_{\beta 100} & S_{\gamma 100} & S_{\delta 100} \\
S_{\alpha 220} & S_{\beta 220} & S_{\gamma 220} & S_{\delta 220} \\
S_{\alpha 333} & S_{\beta 333} & S_{\gamma 333} & S_{\delta 333}
\end{array}\right|
$$

up to division of the second, third, last row by $S_{11}, S_{222}, S_{3333}$ respectively. 
All the entries of this determinant are $4 \times 4$ minors of the matrix :

column index $\left[\begin{array}{cccccccccc}0 & 1 & 2 & 3 & 4 & 5 & \alpha & \beta & \gamma & \delta \\\right.$\cline { 2 - 8 } & $\left.S_{1} & S_{2} & S_{3} & S_{4} & S_{5} & S_{\alpha+3} & S_{\beta+3} & S_{\gamma+3} & S_{\delta+3} \\ \cdot & S_{0} & S_{1} & S_{2} & S_{3} & S_{4} & S_{\alpha+2} & S_{\beta+2} & S_{\gamma+2} & S_{\delta+2} \\ \cdot & . & S_{0} & S_{1} & S_{2} & S_{3} & S_{\alpha+1} & S_{\beta+1} & S_{\gamma+1} & S_{\delta+1} \\ \cdot & . & . & S_{0} & S_{1} & S_{2} & S_{\alpha} & S_{\beta} & S_{\gamma} & S_{\delta}\end{array}\right]$

Designating a minor by the sequence indexing its columns, the determinant to study is

$$
\left|\begin{array}{llll}
{[012 \alpha]} & {[012 \beta]} & {[012 \gamma]} & {[012 \delta]} \\
{[013 \alpha]} & {[013 \beta]} & {[013 \gamma]} & {[013 \delta]} \\
{[034 \alpha]} & {[034 \beta]} & {[034 \gamma]} & {[034 \delta]} \\
{[345 \alpha]} & {[345 \beta]} & {[345 \gamma]} & {[345 \delta]}
\end{array}\right|
$$

and according to Bazin (cf. [Bl]), factorizes into

$$
\text { [0123] [0134] [0345] [ } \alpha \beta \gamma \delta]
$$

that is, more explicitly, into the product

$$
S_{0000}(\mathbb{A}) S_{1100}(\mathbb{A}) S_{2220}(\mathbb{A}) S_{[\alpha+3, \beta+2, \gamma+1, \delta]}(\mathbb{A}) .
$$

Reintroducing the missing factor $S_{11}(\mathbb{A}) S_{222}(\mathbb{A})$, we obtain the theorem.

QED

As we have said, Padé approximants, formal orthogonal polynomials, continued fraction expansion of formal series are all related to Euclidean division.

As a last example, we illustrate how to write a continued fraction expansion of a series, which one can find in the work of Wronski, Chebyschef or Stieltjes.

Proposition 4 Given an alphabet $\mathbb{A}$, then

$\frac{1}{z} \sigma_{1 / z}(\mathbb{A})=$

$$
\frac{1}{z+S_{1}\left(0-\mathbb{A}^{0}\right)+\frac{S_{2}\left(0-\mathbb{A}^{0}\right)}{z+S_{1}\left(\mathbb{A}^{0}-\mathbb{A}^{1}\right)+\frac{S_{2}\left(\mathbb{A}^{0}-\mathbb{A}^{1}\right)}{z+S_{1}\left(\mathbb{A}^{1}-\mathbb{A}^{2}\right)+\frac{S_{2}\left(\mathbb{A}^{1}-\mathbb{A}^{2}\right)}{\ddots}}} .}
$$

Proof. The validity of such expansion amounts to the recursions (3)

$$
\sigma_{1 / z}\left(\mathbb{A}^{k}\right)=\left(1+\frac{1}{z} S_{1}\left(\mathbb{A}^{k-1}-\mathbb{A}^{k}\right)\right) \sigma_{1 / z}\left(\mathbb{A}^{k}\right)+S_{2}\left(\mathbb{A}^{k-1}-\mathbb{A}^{k}\right) \frac{1}{z^{2}} \sigma_{1 / z}\left(\mathbb{A}^{k+1}\right)
$$

with which we began this text.

\section{References}

[Bl] B. Leclerc, On identities satisfied by minors of a matrix, Advances in Maths. 100 (1993)101-132.

[LP] A. Lascoux, P. Pragacz, Sylvester's formulas for Euclidean division and Schur multifunctions, Max Planck Institut Preprints (2001). 